\title{
The Optimization Model of Runway and Gate Assignment
}

\author{
Nahry ${ }^{1}$, Tjahjono, T. ${ }^{1}$, and Satiti, Y.J. ${ }^{1}$
}

\begin{abstract}
This paper is aimed to develop the optimization model of time slot utilization for both runway and apron gate of airport system. The model considers the objectives of airline company to minimize flight-taxiing-time and waiting-time for both landing and take-off. The optimization makes use of Network Representation (NR) in the form of two bipartite networks in order to transform the problem of runway and apron gate assignment into the network flow problem. Maximum Matching and Minimum Cost Flow on NR are the core of the model. An illustrative example is presented. The result shows that the model could increase both runway and apron gate capacities to as close as their theoretical capacities. Compared to the common practice which concerns only; the nearest available runway slot time, behind the scheduled time, and the nearest location of available gates to serve the flight, the model could reduce the total taxiing and waiting time.
\end{abstract}

Keywords: Apron gate time slot, network representation, minimum cost flow, maximum matching, Runway time slot.

\section{Introduction}

The rapid growth of air transport demand has resulted in the need of the airport authority to maximize the utilization of its existing facilities. The utilization of runway, taxiway, and apron gate is one of the major roles in airport management. The airport authority is compelled to utilize those facilities efficiently so as the theoretical airfield capacity could be fully utilized while the costs imposed to the airlines as well as the airport authority itself could also be minimized. Theoretical airfield capacity is the principal and most fundamental measure of the capacity of runway system. It indicates the average number of movements that can be performed on the runway system in one hour in the presence of continuous demand, while adhering to all the separation requirements imposed by the air traffic management system [1]. In many cases the capacity of runway system in practice (practical capacity) could not be attained due to the uncertainty and complexity of the runway assignment. On the other hand, the capacity of taxiway systems depends greatly on local conditions and the geometric configuration of each airport. The capacity of the taxiway system of major airports almost always exceeds the capacity of the runway system by a considerable margin [1].

\footnotetext{
1 Department of Civil Engineering, Faculty of Engineering, Universitas Indonesia, INDONESIA

Email:nahry@eng.ui.ac.id, tjahjono@eng.ui.ac.id, ties_tisan@yahoo.co.id
}

Note: Discussion is expected before November, $1^{\text {st }} 2013$, and will be published in the "Civil Engineering Dimension" volume 16, number 1, March 2014

Received 17 April 2012; revised 08 July 2013; accepted 10 August 2013
The static capacity of apron is the number of aircraft that can be stationed at the apron at any particular instant, while dynamic capacity indicates the number of aircraft that can be served at the apron per unit of time. The dynamic capacity depends strongly on the gate blocking times [1].

The utilization of runway and apron gate is closely related to the concept of blocking times called time slot allocation. The term of time slot actually refers to the time allocated to the airlines to land and takeoff at an international airport [2]. Hence, the time slot is distinguished to runway time slot and gate time slot. For simplicity, we use "slot" to represent "time slot". The expression of international airport is merely due to its tendency of having high traffic volume which needs special attention for time slot allocation. However, in this research, such definition is extended to the issue of utilization of apron gate and it covers also national airport.

Runway slot concerns to the activities related to the flight landing and take-off, i.e. time at which the aircraft is pushed back to the time of aircraft leaving the runway (for take-off) or vice versa (for landing). The increment time of a runway slot (i.e. total time dedicated to one slot of runway use) depends on the ability of the airport authority to manage the whole process of flight take-off and landing. The lesser the increment of runway slot, the more the airfield capacity of the airport, that is the more flight could be handled in a certain period. Obviously, such situation needs an advanced airport management, particularly of air traffic control system, to guarantee the flight safety while the economical advancement could also be achieved due to time efficiency of landing and take-off process. 
Gate slot is related to the ground activity of the flight departure or arrival, from the time at which aircraft stop at the gate for unloading passenger/baggage to the time at which the aircraft is ready to be departed for the next flight.

Most of the previous researches regarding the time slot focused to the strategic level of time slot assignment. The researches were focused on the policies regarding the slot allocation for airlines in the international airport [1,3,4]. Madas [4] developed a methodological framework for multi-criteria evaluation and selection of the most compatible slot allocation strategy with respect to policy criteria and priorities in various airport setting. Zografos [5] studied the operational side of slot allocation, and developed a model implementing the existing European Union/International Air Transport Association rules, operational constraints, and coordination procedures. Its ultimate objective is to better accommodate airlines' preferences at coordinated airports through the minimization of the difference between the requested and the allocated slot times.

This research is aimed to develop an optimization model which can be applied by the airport authority to assign the runway and gate slots to the flights during certain period of time. This research focuses on the operational side of the runway and gate assignment, based on the right held by the airlines and the actual condition at the time of flight departure or arrival. The optimization is aimed to maximize the runway and gate utilization, and at the same time minimizes the taxiing time, as well as waiting time for landing and take-off. Design of runway and gate slot now becomes more important, particularly for the airport with high air traffic demand, since the ratio of the number of runway or gate to the number of flights scheduled diminished, so the task of runway and gate assignment becomes more complex.

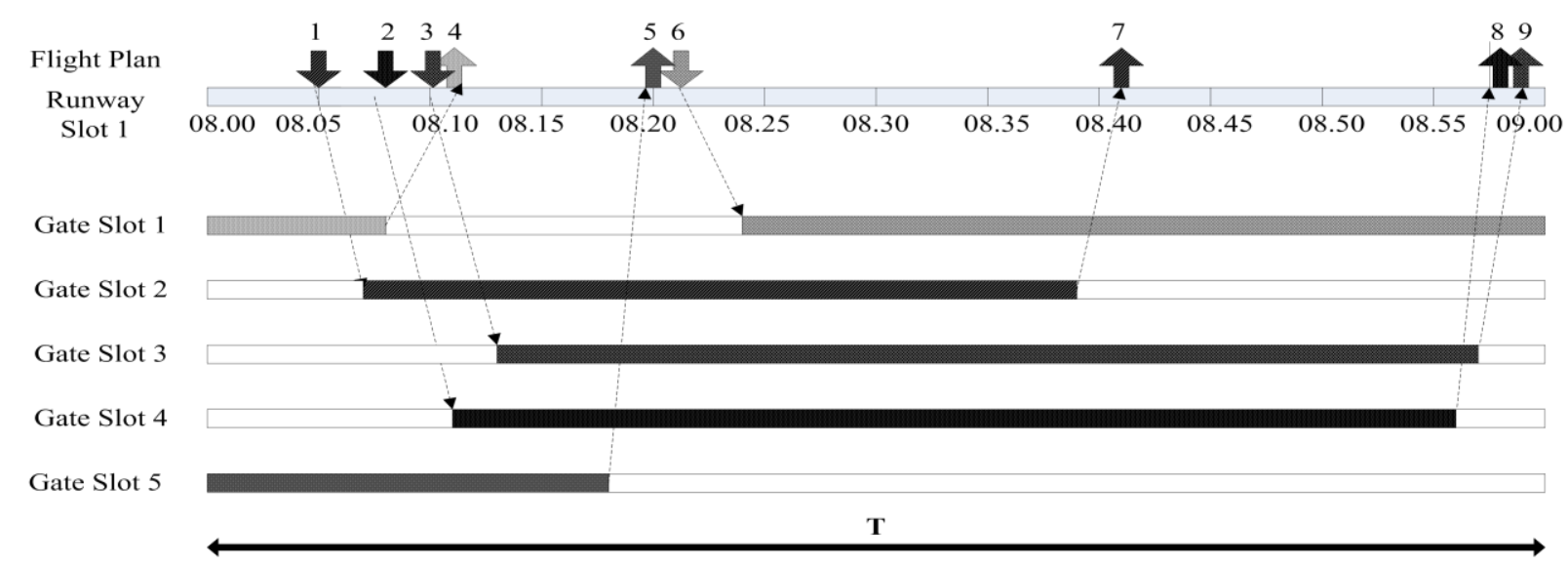

Figure 1. The Representation Diagram of Runway and Gate Assignment Problem

\section{Model Development}

The runway and gate assignment problem will be described through the following hypothetical case. Flight plan of an airport indicates nine scheduled flights between 08.00-09.00 (Table 1).

Table1. Flight Plan

\begin{tabular}{cccc}
\hline No. & Time & Flight & Remarks \\
\hline 1. & 08.05 & GIA 145 & Arrival \\
2. & 08.08 & BTV 047 & Arrival \\
3. & 08.10 & LNI 041 & Arrival \\
4. & 08.11 & AXN 842 & Departure \\
5. & 08.20 & GIA 131 & Departure \\
6. & 08.21 & BTV 032 & Arrival \\
7. & 08.41 & GIA 146 & Departure \\
8. & 08.58 & BTV 048 & Departure \\
9. & 08.59 & LNI 042 & Departure \\
\hline
\end{tabular}

In order to assign those flights to the runway and apron gate, it is required to manage the facilities, i.e the runway, taxiway as well as apron gates in certain way so as the operational cost of the aircraft as well as the airport could be minimized. In this case, the operational cost is represented by the operational time and it is denoted by the waiting time at the taxiway before take-off or waiting time in the air before landing, and taxiing time. The process of runway and gate assignment is described through the diagram of runway and gate assignment (Figure 1). At the upper row of the diagram, some arrows are used to indicate the departure and arrival of scheduled flights as stated in flight plan of Table 1. The nine flights are set to the respective time of the diagram in which the horizontal axis is respected to time.

Figure 1, the representation diagram of runway and gate assignment problem Minimum Cost Flow (MCF) problem is network flow problem which is aimed to determine a least cost shipment of a commodity through a network in order to satisfy 
demands at certain nodes from available supplies at other nodes [7].

Figure 2 denotes the rationale of development of NR of runway and gate assignment. Since the network of NR consists of nodes and links, the development of $\mathrm{NR}$ is initialized by defining the nodes of the NR. Every flight arrival and departure stated in flight plan is set as one node in NR. In the illustrative case, node 101 is assigned to represent flight no.1 (arrival) on the flight plan, and node 109 as flight no. 9 (departure). Such nodes are designated as Flight Nodes.

Every increment of runway slot is designated as Runway Node (e.g. node 201-213) and every increment of gate slot is designated as Gate Node (e.g. node 301-312). Figure 2 indicates the position of the 3 types of nodes, which are named with the initial number 1, 2, and 3 for flight nodes, runway nodes, and gate nodes, respectively. Such naming rule is applied only for the sake of simplicity of explanation in this paper. It is not a compulsion. Every flight node holds the following attributes: flight node time (i.e. departure or arrival time), type of movement (arrival or departure), Remaining Over Night (RON) label, Slot Right label (Grandfather Right) and ground time (including loading/unloading time). Every runway node holds runway node time and runway number, while every gate node holds the following: attributes gate node time, label of passenger loading system, distance from runway to gate, and Slot Right label (Grandfather Right). Those attributes are the basic variables of the model. Definitely, they may be modified in order to represent the actual variables which are considered in the model.
The next step is to develop the network of NR problem. Two bipartite networks are developed. The first bipartite network (BN-1) consists of Flight Node sub network and Runway Node sub network, while the second one (BN-2) consists of Used Runway Node sub network and Gate Node sub network (Figures 3 and 4). The links which connect the two sub networks of $\mathrm{BN}-1$ are designated to represent the feasible connections between flight schedule and runway slot. On the other hand, the links between the two sub networks of $\mathrm{BN}-2$ are designated to represent the feasible connections between the use of runway and the apron gate. The time consequences of those connections, such as taxiing time and waiting time, are applied as the attributes of the links (i.e. unit cost of link).

The runway and gate assignment process which satisfy the mathematical programming of Equation 1 is represented by the problem of searching the maximum matching of each $\mathrm{BN}$ with the minimum cost. In this case, one matching is analogous to placing one flight node to one and only runway node of Figure 2 or placing one used-runway node to one and only gate node. The notion of matching is chosen since in a certain period a runway can handle only one movement, and in addition one gate also can handle only one aircraft. Then, the maximization of number of matching is a representation of maximization of slot utilization. Since MCF problem holds the same objective with the chain of assignment problem, it will be applied in searching the maximum matching in order to guarantee the cost consequence of the searching to be minimum. Eventually, it can be concluded that the maximum matching with minimum cost in both $\mathrm{BNs}$ will represent runway and gate assignment tasks. The
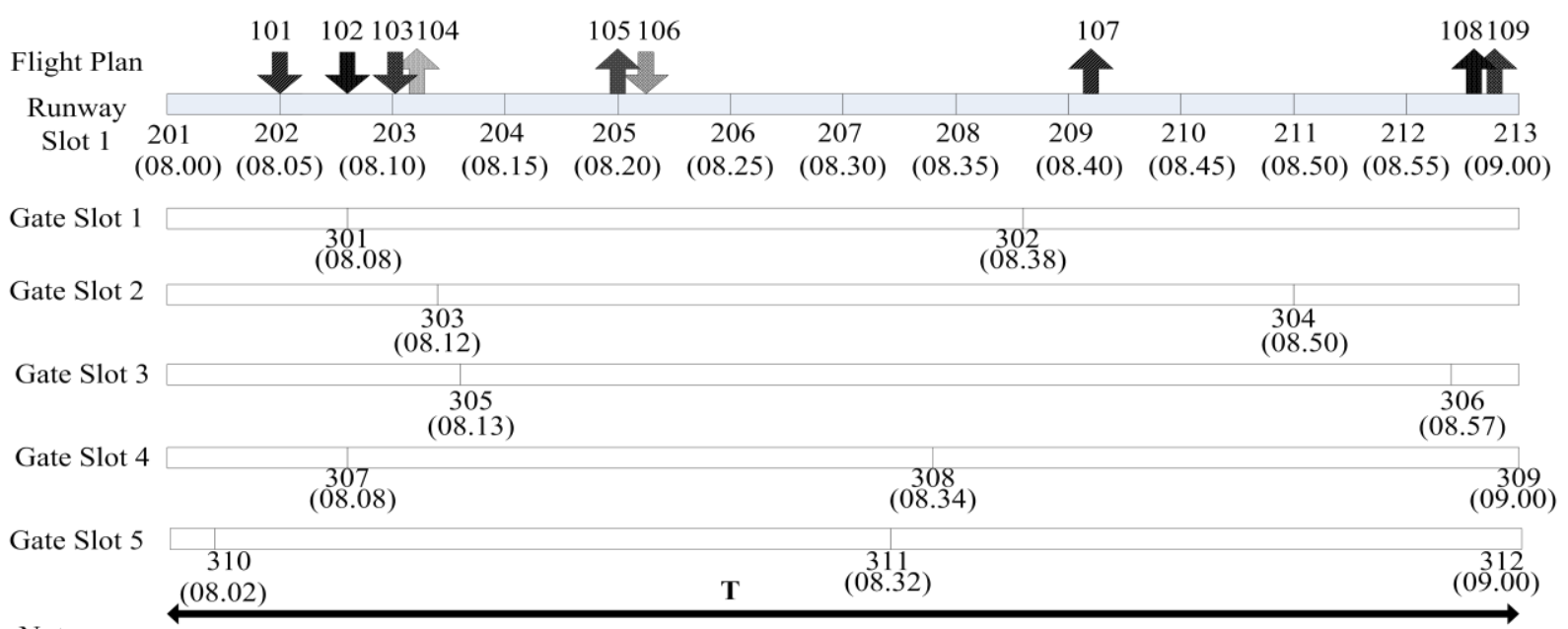

Notes :

Node $101 \sim 109$ : Flight Node

Node $201 \sim 213$ : Runway Node

Node $301 \sim 312$ : Gate Node

Figure 2. The Development of Components of Network Representation 
development of Sub Model 1 and Sub Model 2 will be described in the following sections.

\section{Sub Model 1 Development}

The algorithm of Sub Model 1 can be described as follows:

Step 1: Set the runway time slot during time period $T$

Step 2: Develop the bipartite network of Network Representation

Step 2a: Define the subset $\left\{X^{\prime}\right\}$ and $\left\{X^{\prime \prime}\right\}$, as well as their associated attributes, where $X^{\prime}$ is the set of Flight Nodes and $X^{\prime \prime}$ is the set of Runway Nodes

Step 2b: Connect set $\left\{X^{\prime}\right\}$ and $\left\{X^{\prime \prime}\right\}$ with the all feasible links, with the following rules:

- For departure flight: $-\alpha_{2} \leq t f n_{f}-t r n_{r} \leq \alpha_{2}$, $\forall f \in F, r \in R$

- For arrival flight: $-\alpha_{1} \leq t f n_{f}-t r n_{r} \leq \alpha_{1}, \forall f \in$ $F, r \in R$ where:

$\alpha_{1}$ : Maximum allowable time gap to shift the flight arrival time

$t r n_{r}$ : Time attribute of runway node - $r$

$t f n_{f}$ : Time attribute of flight node $-f$

$\alpha_{2}$ : Maximum allowable time gap to shift the flight departure time

$R$ : Set of runway nodes

$F \quad$ : Set of flight nodes

Step 3: Value all the links with their unit cost with the following rules:

- For departure flight: If $t f n_{f} \geq t r n_{r}, c_{f r}=$ $\alpha_{5}\left(t f n_{f}-t r n_{r}\right)$ where $1<\alpha_{5}<\sim$ If $t f n_{f}<t r n_{r}, c_{f r}=\alpha_{4}\left(t r n_{r}-t f n_{f}\right)$, in which $1<\alpha_{4}<\sim$

- For arrival flight: If $t f n_{f} \geq t r n_{r}, c_{f r}=t f n_{f}-$ $t r n_{r}$

If $t f n_{f}<t r n_{r}, c_{f r}=\alpha_{4} \alpha_{5}\left(t r n_{r}-t f n_{f}\right)$, in which $1<\alpha_{4}<\sim$ and $1<\alpha_{5}<\sim$

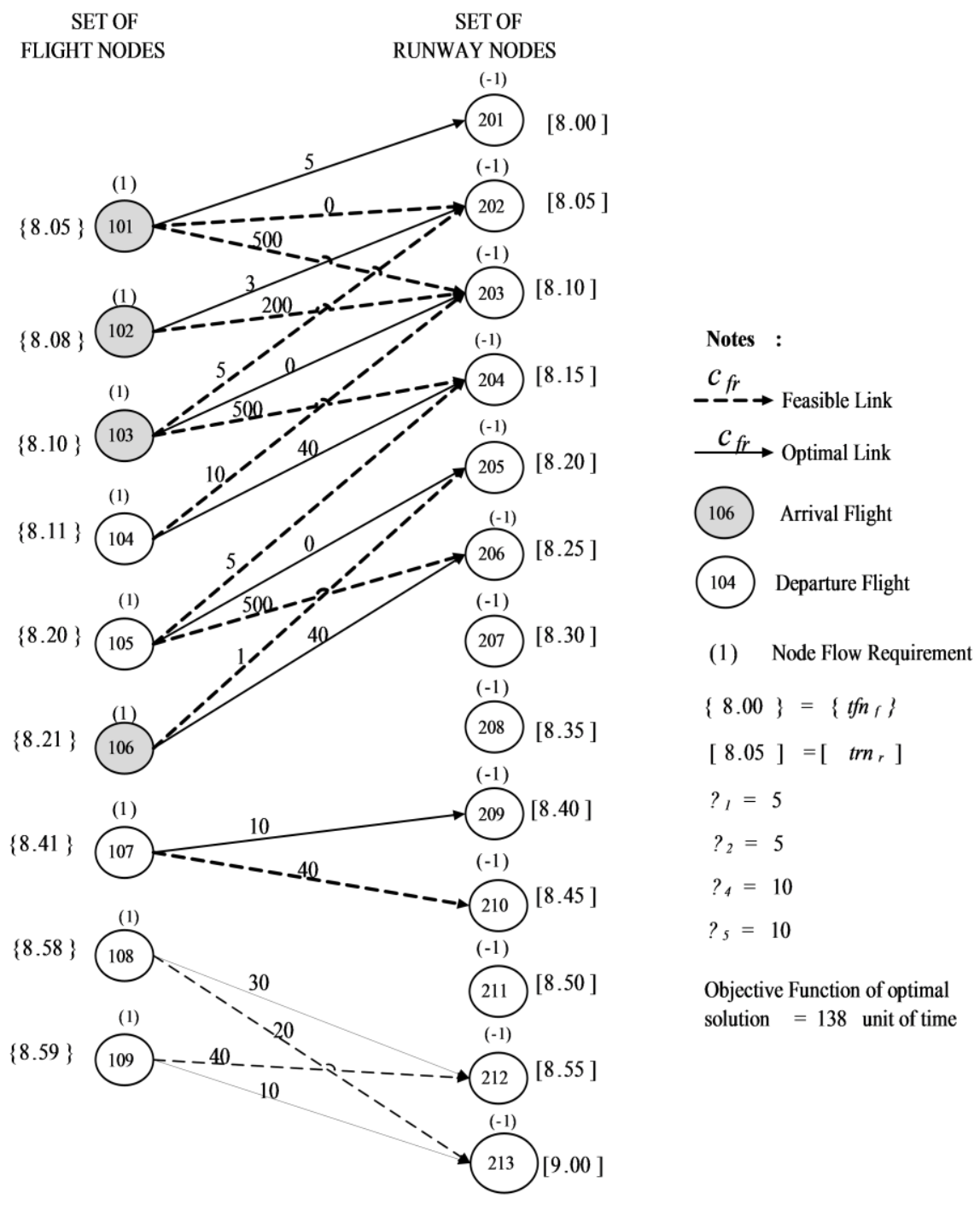

Figure 3. Bipartite Network of Sub Model 1 (BN-1 


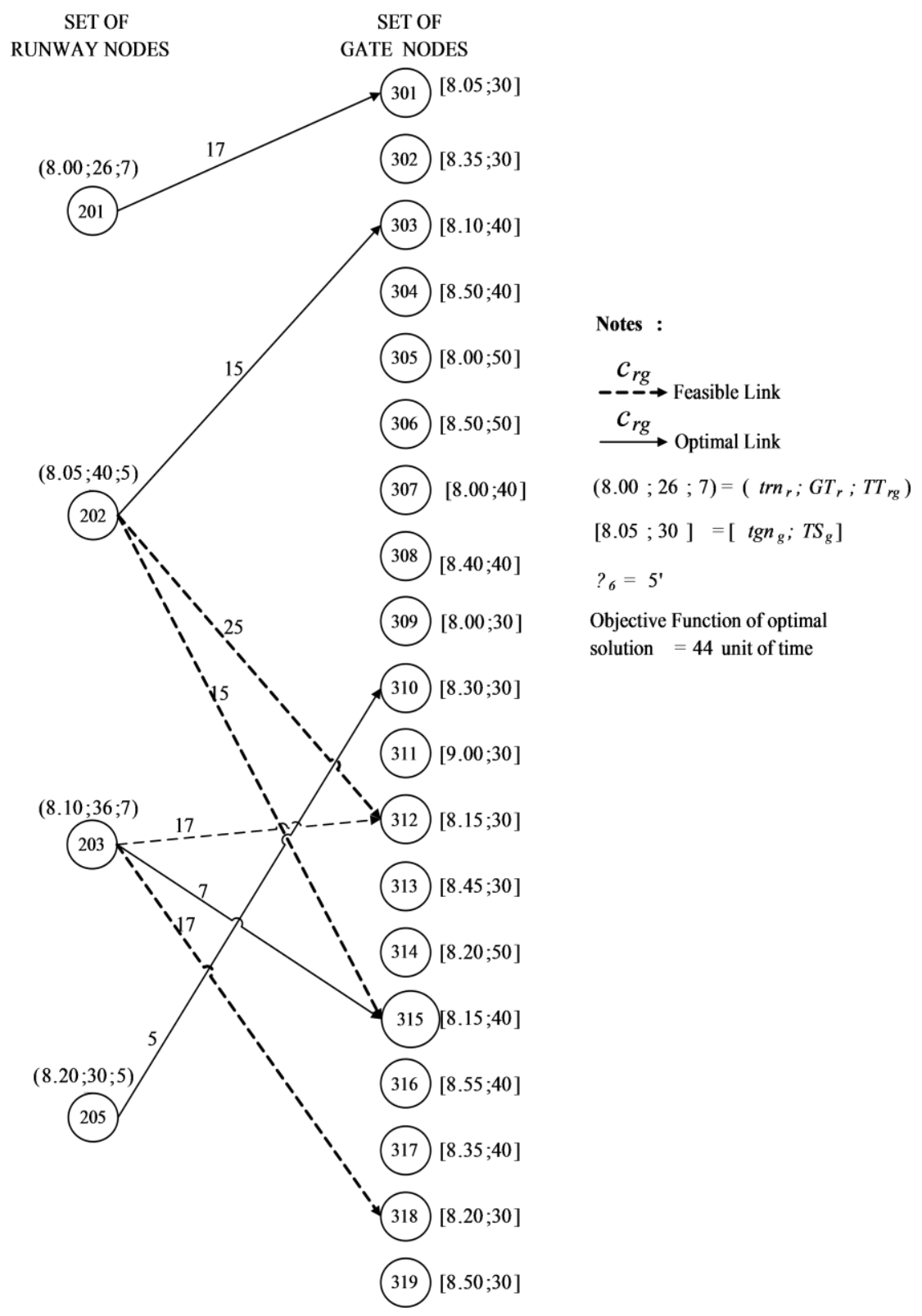

Figure 4. Bipartite Network of Sub Model $2(\mathrm{BN}-2)$

where:

$c_{f r}$ : Unit cost of link between flight node- $f$ and runway node-r

$\alpha_{4}$ : Penalty factor for schedule postponing. It represents the additional cost which should be imposed to flight postponing.

$\alpha_{5}$ : Priority factor dedicated to flight arrival. It shows that runway use priority should be given to the flight arrival in order to reduce airfield delay

Step 4: Set the link capacity as: $\operatorname{Cap}_{f r}=1, \forall f \in F$, $r \in R$; and node flow requirement as: $b_{f}=1, \forall f \in F$ and $b_{r}=-1, \forall r \in R$

Step 5: Find the optimal flow of the BN-1 using the algorithm of MCF
In step $2 b$, feasible links between set of flight nodes and set of runway nodes are set based on the rules formulated in Equations 5 and 6. Equation 5 guarantees the time gap between the flight arrival or departure and the runway slot time under consideration satisfies the allowable time. It is allowed to postpone or advance flight schedule as long as the maximum allowable time $\left(\alpha_{1}\right.$ or $\left.\alpha_{2}\right)$ is not violated. It is aimed to utilize the runway as much as possible.

Further, in step 3 the unit cost of each feasible link $\left(C_{f r}\right)$ is calculated, representing the time difference between the arrival or departure time and the runway slot time. The bigger the difference, the bigger the unit cost would be. It is reasonable that an attempt to optimize the runway assignment must be 
aimed to minimize waiting time of either at ground or in the air. Hence, searching of pair of flight node and runway node which gives the minimum cost is relevant to the effort of minimizing ground or airfield waiting time. Since the effort to postpone the flight arrival/departure schedule is more discouraged than advance it, parameter $\alpha_{4}$ is used in Equations 8 and 10 to multiply link unit cost and then the postponing could be avoided as much as possible. Moreover, since the postponing of flight arrival (in the air) is more costly than the one of flight departure (at ground), this notion is represented in the form of parameter $\alpha_{5}$ in Equations 7 and 10. Further, step 4 shows that each link of NR could only be passed by one unit of flow, and surely it is relevan to the effort of searching the maximum matching of $\mathrm{BN}-1$. In addition, the node flow requirement which is set as 1 for all the origin nodes and -1 for all the destination nodes of BN-1 enables all the flight nodes to sent flow to runway nodes as long as there exists link between both sets of nodes. Finally, in step 5 the assignment process of $\mathrm{BN}-1$ can be carried out using any kind of algorithm which is based on MCF concept. Primal Dual algorithm is one of the algorithms which could be applied to solve this problem [7]. From the solution of MCF, the links which bear one unit of flow constituted matching between flight node and runway node, representing the time of using the runway of each scheduled flight.

The optimal solution of MCF problem for case of Table 1, as well as the feasible links of BN-1 is shown in Figure 3. The chain of assignment of this optimal solution is depicted in Figure 5. In Figure 5, the first flight which is initially scheduled at 08.05 is moved forward to 08.00 and landing by using runway slot 201. Due to such flight movement, flight no. 2 could also be moved forward to runway slot 202. Further, though the flight plan shows that flight no. 5 is scheduled earlier than flight no. 6 , the final assignment shows that flight no. 5 (as departure flight) is postponed 5 minutes in order to give priority to flight no. 6 (as arrival flight) to land one minute ahead. The remaining flights could be explained similarly. This optimal solution gives the objection function as 138 unit of time. When the runway assignment is solved by the existing mechanism, i.e. each flight is assigned to the nearest time behind the scheduled time, the objective function attains 2500 unit of time. In such case, all flights (except flight no.1) are postponed, and even flight no. 9 must be moved to the next period. Further, the output of Sub Model 1 is applied as the input of Sub Model 2.

\section{Sub Model 2 Development}

The algorithm of Sub Model 2 can be described as follows:

Step 1: Set the apron gate time slot during time period $T$

Step 2: Place the existing flights, i.e flights which have occupied the gate before $T$ started, in the respected gate time slot.

Step 3: Develop the bipartite network of Network Representation

Step 3a: Define the subset $\left\{X^{\prime}\right\}$ and $\left\{X^{\prime \prime}\right\}$, as well as their associated attributes, where $X^{\prime}$ is the set of runway nodes and $X^{\prime \prime}$ is the set of gate nodes, with the following rule: the runway node associated to the departure is not included in sub set $\left\{X^{\prime}\right\}$ for all flights with departure and arrival time in period $T$.

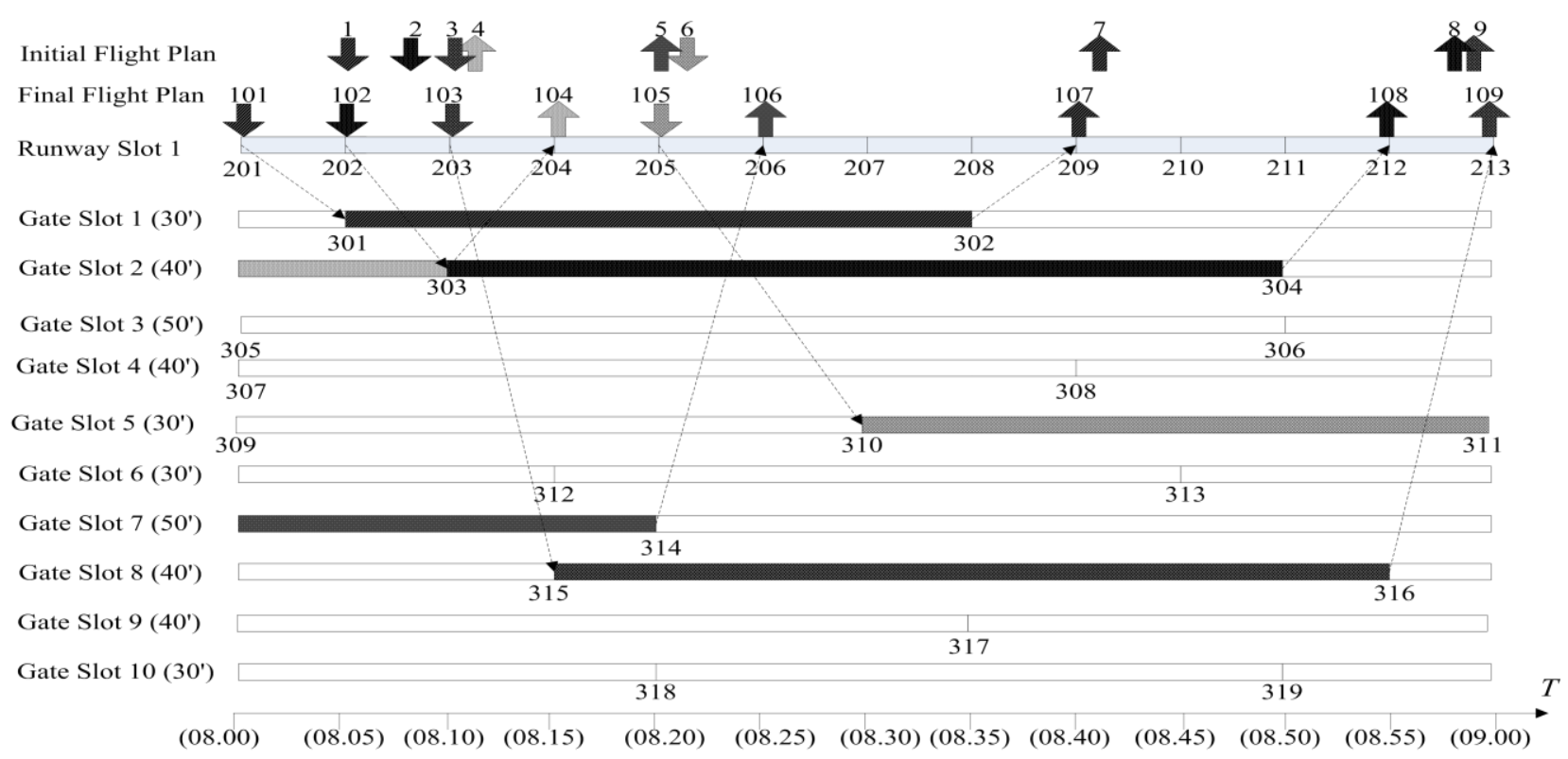

Figure 5. Chain of Runway and Apron Gate Assignment 
Step 3b: Connect set $\left\{X^{\prime}\right\}$ and $\left\{X^{\prime \prime}\right\}$ with all feasible links, with the following rules:

- For arrival flight: $\left(T T_{r g}-\alpha_{6}\right) \leq t g n_{g}-t r n_{r} \leq$ $\left(T T_{r g}+\alpha_{6}\right), \forall r \in R, g \in G$ $\left(G T_{r}-2 \alpha_{6}\right) \leq T S_{g} \leq\left(G T_{r}+2 \alpha_{6}\right), \forall r \in R$, $g \in G$

- For departure flight with initial position remoted: $\left(T T_{g r}+G T_{r}-\alpha_{6}\right) \leq t r n_{r}-t g n_{g} \leq\left(T T_{g r}+\right.$ $\left.G T_{r}+\alpha_{6}\right), \forall r \in R, g \in G$

$\left(G T_{r}-2 \alpha_{6}\right) \leq T S_{g} \leq\left(G T_{r}+2 \alpha_{6}\right), r \in R, g \in G$ (14)

- Such rules are not valid for flights which have occupied the gates before $T$ started. In this case, the only link (for each flight) is between its runway slot and the associated gate slot in use.

where:

$t r n_{r}$ : Time attribute of runway node $-r$

$T T_{r g}$ : Taxiing time from runway associated to node $-r$ to apron gate associated to node $-g$ (including the average time for clearing the runway area, i.e. two minutes)

$T T_{g r}$ : Taxiing time from apron gate associated to node $-g$ to runway associated to node $-g$ (including the average time for clearing the runway area, i.e. two minutes)

$G \quad:$ Set of gate nodes

$t g n_{g}$ : Time attribute of gate node $-g$

$\alpha_{6}:$ Maximum allowable time gap to shift the time of gate occupation

$T S_{g}$ : Time increment of gate slot associated to node $-g$

$G T_{r}$ : Ground time of flight associated to node $-r$ (start from the aircraft parked at the apron to the aircraft leave the apron gate)

$R \quad$ : Set of runway nodes

Step 4: Value all links with their unit cost with the following rules:

$c_{r g}=t d r_{r}-t a r_{r}-T S_{g}+T T_{r g}, \forall r \in R, g \in G$

For the flights with lengthy ground time and/or remoted aircraft, it is set the following rule:

$c_{r g}=T T_{r g}, \forall r \in R, g \in G$

where:

$c_{r g}:$ Unit cost of link between runway node $-r$ and gate node-g

tar $_{r}$ : Time arrival associated to runway node $-r$

$t d r_{r}$ : Time departure associated to runway node $-r$

Step 5: Set the link capacity as $\operatorname{Cap}_{r g}=1, \forall r \in R$, $g \in G$, and node flow requirement $b_{r}=1, \forall r \in R$ and $b_{g}=-1, \forall g \in G$

Step 6: Find the optimal flow of the BN-2 using the algorithm of MCF

In step 3a, the member of set of used-runway nodes and gate nodes is identified. For the aircraft whose arrival and departure time fall within period $T$ and is kept staying at the gate (not remoted), its departure flight is not considered in the gate assignment. The gate associated to such departure flight must be the same to the gate of its arrival. In step 3b, feasible links between set of runway nodes and of gate nodes are established based on the rules formulated in Equations 11 to 14. Equation 11 and 13 depict that the time gap between the arrival or departure time (at runway position) and the time associated to gate slot under consideration must be within the allowable time. In this case, flight is allowed to wait shortly before the gate opened or shrink its gate occupation time as long as within permissible time $\left(\alpha_{6}\right)$. It is aimed to increase the utilization of gate. In order to accommodate other practices of using the gate, (e.g. grand father right, existence of aerobridge, etc), rules in step $3 \mathrm{~b}$ may be modified. However, the basic rule is that feasible links could be established only when they could satisfy the operational constraints. Further, since the increment time of gate slot may be different one from another, then Equations 12 and 14 are aimed to check if the ground time required by the aircraft appropriates with the time provided by gate slot, i.e. within allowable gap time $\left(\alpha_{6}\right)$. For flight which has occupied gate before $T$, there is only one feasible link, that is the link between runway node associated to such flight and gate node associated to the occupied gate.

Moreover, in step 4 the unit cost of all feasible links $\left(C_{r g}\right)$ are calculated. It represents the difference between the gap between time of arrival and departure and the increment of gate slot under consideration, plus the taxiing time. The bigger the difference, the bigger the unit cost would be. In addition, the bigger the taxiing time to the apron gate or to the runway, the bigger the unit cost would be. From this point, it is reasonable that an attempt to optimize the apron gate assignment is relevant to seeking the pair of runway and gate nodes which gives the minimum cost, that is the one that synchronize the ground time and the increment time of gate slot at the most, as well as the one with the minimum taxiing time. Further, step 5 and step 6 are carried out in the similar ways with step 4 and step 5 of Sub Model 1. In the optimal solution, the links which bear one unit of flow constituted matching between used-runway node and gate node, representing time of gate occupancy of each scheduled flight. The optimal solution of MCF for case of Table 1, as well as the feasible links of BN-2 is shown Figure 4. The chain of assignment is depicted in Figure 5. The optimal solution of Sub Model 2 gives the objective function as 44 unit of time. 
In case of Table 1 , based on the gate assignment before $T$, the departure of flight no. 4 (flight node 104) and flight no. 5 (flight node 106) have been served by gate no. 2 and 7 , respectively (Figure 5). In addition, the departure of flights no. 7, 8, and 9 are not included in gate assignment since the pair of those flights, i.e. the arrival of flight no. 1,2 , and 3 , is within period of $T$. Hence, only runway node 201, 202, 203, and 205 will be included in the gate assignment (Figure 4).

The optimal solution of Sub Model 2 shows that flight no. 3 (node 203) will be served by gate 8 (node 315) even though gate 6 and 10 (node 312 and 318) are also feasible to be chosen. It can be explained that since the gate assignment considers the appropriateness between the ground time and the increment time of gate slot, so the best choice is gate 8 which offers 40 minutes of gate slot. The 30 minute gate slot of gate 6 and 10 may cause inconvenience to the passenger due to the reduced ground time. Further, flight no. 2 (node 202) will be served by gate 2 (node 303) even though gate 6 and 8 (node 312 and 315) are also feasible. Here, the use of either gate node 312 or 315 will create similar penalty, since the gate time slot is not suitable enough with the ground time required compared to gate node 303 . The slot of gate 6 (node 312) makes flight no. 2 waiting before gate occupancy and also waiting for the take-off outside the gate. Further, slot of gate 8 (node 315 ) is treated in almost similar way. When those rules are not considered in the assignment and it takes into account only the nearest available slots to the flight schedule, the objective function attains 54 unit of time.

\section{Conclusion}

The proposed model could be used to optimize the use of runway and apron gate by considering waiting time for both landing and take-off as well as taxiing time. However, since the increment time of runway and gate slot are set by the airport authority, and they refer to the theoretical operational capability of the airport to handle landing and take-off activities as well as ground handling at the apron gates, the model concerns to the increase of practical capacity instead of theoretical one. The model may reduce the complexity of the assignment and it may lead to the increase of practical capacity and the decrease of taxiing and waiting time. Further researches are required to consider the dynamic of flight schedule during time $T$, so the model could represent the problem more realistically.

\section{References}

1. Neufville, R. and Odoni, A., Airport Systems. Planning, Design and Management, McGraw Hill, USA, 2003.

2. Abeyratne, R.I.R., Management of Airport Congestion through Slot Allocation, Journal of Air Transport Management, 6, 2000, pp. 29-41.

3. Madas, M.A. and Zografos, K.G., Airport Slot Alocation: From Instrument to Strategy, Journal of Air Transport Management, 12, 2006, pp. 53-62.

4. Madas, M.A. and Zografos, K.G., Airport Capacity vs Demand: Mismatch or Mismanagement?, Transportation Research, Part A, 42, 2008, pp. 203-226.

5. Zografos, K.G., Salouras, Y., and Madas, M.A., Dealing with the Efficient Allocation of Scarce Resources at Congested Airports, Transportation Research, Part C, 21, 2012, 244-256.

6. Minieka, E., Optimization Algorithms for Networks and Graphs, Marcel Dekker Inc., NewYork, 1978.

7. Ahuja, R.K., Magnanti, T.L., and Orlin, J.B., Network, Prentice Hall, New Jersey, 1993. 